\title{
Estructura y composición de encinares naturales en las Alturas de Pizarras del municipio de Viñales, Pinar del Río, Cuba
}

\section{Structure and composition of natural holm oaks in the Slate Altitude from Viñales Municipality, Pinar del Río, Cuba}

\author{
Modesto González-Menéndezl*, Yatsunaris Alonso-Torrens², Isyoel Urrutia-Hernández', Yaumara Miñoso³, \\ Isabel Santana ${ }^{4}$, Jesús Luis-Suárez' y Carlos Manuel González-Torres ${ }^{5}$
}

Universidad de Pinar del Río. Centro Universitario Municipal Viñales, Viñales, Pinar del Río, Cuba.

2 Universidad de Pinar del Río. Facultad de Forestal y Agronomía. Departamento Forestal, Pinar del Río, cuba. yatsunaris@upr.edu.cu

\author{
3 Instituto de Investigaciones Agroforestales. Esta- \\ ción Experimental Agro- Forestal Viñales (EEAF). \\ Pinar del Río, Cuba. yaumara@vinales.inaf.co.cu \\ ${ }^{4}$ Instituto de Investigaciones Porcinas de Cuba. \\ isantana@iip.co.cu
}

\author{
5 Flora y Fauna, Unidad Empresarial de Base (UEB). El \\ Rosario, Viñales, Pinar del Río, Cuba. \\ * Autor de correspondencia. \\ modestogonzalez208@gmail.com
}

\section{RESUMEN}

La investigación fue realizada en encinares de las Alturas de Pizarras de la Empresa Agroforestal La Palma y de la Estación Experimental Agroforestal Viñales, con el objetivo de determinar la estructura y composición de los encinares naturales. Se seleccionaron dos sitios diferentes en cuanto a nivel de intervención del hombre. Se levantaron 10 parcelas cuadradas $\left(100 \mathrm{~m}^{2}\right)$, cinco en cada una de las áreas incluidas en el estudio, distribuidas de forma aleatoria simple. Se calcularon las diversidades alfa y beta, se evaluó la estructura horizontal mediante el índice de importancia ecológica y la distribución por clases diamétricas, se describió la estructura vertical a través de la riqueza de especies en cada estrato así como su posición sociológica. La riqueza de especies evidenció una alta diversidad florística asociada al encinar natural. Se identificaron 38 especies asociadas a los encinos, agrupadas en 25 familias, siendo las familias Melastomataceae con cuatro especies y Fabaceae, Rubiaceae y Sapindaceae con tres especies, las mejor representadas. Se comprobó que el nivel de intervención silvícola y pastoril con cerdos tuvo una marcada influencia en la estructura y composición de los encinares naturales. Se determinó que Quercus cubana A. Rich, como la especie dominante y de mayor importancia ecológica en esta formación; posee una presencia irregular en la estructura de este bosque, convirtiéndola en vulnerable ante disturbios naturales y de actividades antrópicas potenciales, evidenciando una habilidad competitiva débil. Los resultados obtenidos, constituyen un referente para el manejo de esta especie endémica del occidente de Cuba.

PALABRAS CLAVE: estructura horizontal, clases diamétricas, posición sociológica, disturbios naturales, intervención humana, rebaño porcino.

\section{ABSTRACT}

The investigation was carried out in the holm oaks in the Slate Altitude in the Agroforestry La Palma and in the Agroforestry Experimental Station of Viñales Municipality, with the objective to determine the structure and composition of natural oaks. Two different places were layed out regarding a level of man intervention. There were established 10 square plots $\left(100 \mathrm{~m}^{2}\right)$, five in each of the included in the areas in the study, in way simple aleatoia. Alpha and beta diversity was determined; the horizontal structure was evaluated by calculating the index of ecological importance and the distribution by diameter classes. Also, it was described the vertical structure through the richness of species in each stratum as well as its sociological position. The richness of the species proved a high floristic diversity associated to the oaks. There were identified 38 species associated to the oaks, grouped in 25 families, where the Melastomataceae family with four species and Fabaceae, Rubiaceae and Sapindaceae with three species is the most represented. It was proved that the silvicola management and pastoral of the pig livestock level had strong influence in the structure and composition of the natural oaks. It was determined that the Quercus cubano A. Rich. As dominant specie with the most ecological importance in this formation, it has irregular presence in the structure of this forest, turning to be vulnerable to natural disturbances potential human intervention, showing a weak competitive skill. The obtained results constitute a reference for the management of this endemic specie in the west of Cuba.

KEYWORDS: horizontal structure, diameter classes, sociological position, natural disturbances, man intervention, pig livestock. 


\section{INTRODUCCIÓN}

Los bosques son el reservorio de diversidad biológica terrestre más grande, desempeñan un papel fundamental en la mitigación del cambio climático mundial y contribuyen a la conservación de los suelos y las aguas en muchos ecosistemas frágiles (Organización de las Naciones Unidas para la Alimentación y la Agricultura [FAO], 2014). Algunos autores expresan que el estudio de la composición, estructura y dinámica de un bosque es el punto de partida para la adecuación de criterios, métodos de conservación y recuperación de ecosistemas (Finegan, Palacios, Zamora y Delgado, 2001). Algunos de los cambios pueden percibirse como positivos, ya que favorecen a especies utilizadas como recursos, o crean condiciones favorables para los seres humanos. Según Pickett y White (1985), una perturbación es un suceso discreto en el tiempo que altera la estructura de los ecosistemas, de las comunidades o de las poblaciones.

En la región tropical, los encinares están en peligro de extinción. Muchos están muy degradados y aunque todavía hay algunos individuos grandes y viejos, no existen bosques primarios de encino. La mayoría de los encinares han sido convertidos en potreros para ganado. Una gran parte de los encinares se ven como una sabana de pasto con árboles solitarios y pequeños de encino, frecuentemente asociados con Byrsonima crassifolia, Curatella americana y Gliricidia sepium (Pennington y Sarukhán, 1998).

En Cuba, por las características de su madera, los bosques de encino han sido muy explotados con fines de extracción de madera, para la elaboración de carbón, horcones de casas (para vivienda y para curar tabaco), así como para la construcción de implementos de trabajo de uso doméstico, lo cual provoca que este tipo de vegetación tienda a fases secundarias (González et al., 2016).

Q. cubana A. Rich es la única especie de este género presente en la flora cubana, específicamente en el occidente, crece en asociación con pinares naturales; está reconocida como endémica cubana, limitada solo a Pinar del Río (Izquierdo et al., 2013). Su madera es apreciada por su dureza y durabilidad; por su alto poder calorífico es excelente como dendroenergía. También es empleada en la construcción de implementos de trabajo, horcones para viviendas y casas de curar tabaco entre otros (Izquierdo, Pimentel, \& Márquez, 2013).

Los frutos, comúnmente llamados bellotas, tienen un uso muy difundido en la vida rural; poseen un alto contenido en grasas, lo que constituye un excelente alimento para los cerdos, engordándolos y dándole un gusto peculiar y agradable a su carne (Santana, Abeledo, Ferrer, González yPimentel, 2015).

En la actualidad los encinares presentan una situación crítica, debido al mal manejo (silvícola y sobre pastoreo) a que han estado sometidos en el tiempo, esto ha traído como resultado el envejecimiento y deterioro de las áreas naturales y un crecimiento pobre en plantaciones, trayendo consigo un notable decrecimiento en la producción de frutos y de su calidad, viéndose afectado su uso para ceba de cerdos en comunidades rurales (González et al., 2016).

\section{OBJETIVOS}

Determinar la estructura y composición de encinares naturales en las Alturas de Pizarras del Municipio de Viñales, Pinar del Río, Cuba.

\section{MATERIALES Y MÉTODOS}

\section{Área de estudio}

El estudio se llevó a cabo en la formación montañosa Alturas de Pizarras del centro de Pinar del Río, Cuba, ubicada en la cordillera de Guaniguanico, específicamente en áreas de encinares naturales pertenecientes a la Estación Experimental Agroforestal de Viñales (EEAF) y a la Empresa Agroforestal La Palma, zona esta donde se encuentra $85 \%$ de los encinares naturales de la provincia (Servicio Estatal Forestal [SEF]. 2016). La precipitación promedio anual del área de estudio es de $1765 \mathrm{~mm}$, la temperatura promedio anual de $25{ }^{\circ} \mathrm{C}$. Presenta una temperatura máxima de $28.8^{\circ} \mathrm{C}$ y una mínima de $19.5^{\circ} \mathrm{C}$. Según Hernández, Ascanio, Carrera y Morales (2003), los suelos en donde se realizó el estudio se clasifican como Alíticos, destacándose en ellos el alto contenido de aluminio 
y que el contenido de arcilla es mayor en el subsuelo que en el suelo superficial.

\section{Muestreo}

El estudio de la vegetación se realizó con un muestreo aleatorio simple, estableciéndose parcelas cuadradas de 10 $\mathrm{m} \times 10 \mathrm{~m}$ en un encinar natural poco antropizado, en áreas de la Estación Experimental Agroforestal de Viñales y en un área productiva más antropizada, de la Empresa Agroforestal La Palma (Tabla 1).

\section{Diversidad alfa $(\alpha)$}

La diversidad (alfa) de especies forestales en cada encinar fue estimada mediante la riqueza de especies (Magurran, 1989). Se determinaron además las curvas suavizadas de acumulación de la riqueza observada (curvas de rarefacción basadas en muestras) para la vegetación presente en cada encinar mediante el software Estimates versión 9.0.0 (Colwell, 2011).

Se confeccionaron los listados florísticos correspondientes, con los cuales fue posible calcular los índices de diversidad de especies de Margalef, ShanonWeaner y Berger-Parker, utilizando las ecuaciones 1, 2 y 3 (Moreno, 2001).

$$
D M g=(S-1) / \ln N
$$

Donde:

$D M g$ : Índice de Margalef

$S$ : Número de especies

$N$ : Número total de individuos

$$
H^{\prime}=-\sum p i * \ln p i
$$

Donde:

$H$ : Índice de Shannon-Wiener

$p_{i}$ : Valor de importancia, calculado mediante la ecuación 3

$$
P i=n i / N
$$

Donde:

$N i$ : Número de individuos de la especie

$N$ : Número total de individuos de la muestra

La determinación de los diferentes índices de diversidad de especies se realizó utilizando el programa BioDiversity Professional (McAleece, Gage, Lambshead y Patterson, 1997). Para la nomenclatura de las especies presentes se siguieron los criterios de Sánchez (2007) y Acevedo y Strong (2012).

\section{Diversidad beta $(\beta)$}

Con el fin de evaluar la similitud florística entre las parcelas y las poblaciones, se realizó un análisis de conglomerados usando el software PC-ORD (McCune y Mefford, 1999), con la medida de distancia de Sorensen cuantitativo (BrayCurtis) y el método de asociación de los grupos fue el de Ward's (Acosta, Araujo e Iturre, 2006).

\section{Estructura horizontal}

Para señalar la importancia de cada especie en el conjunto, se determinó el índice de valor de importancia ecológica (IVIE), cuyo resultado es la suma de los valores relativos de abundancia o densidad, dominancia y frecuencia de cada especie (Acosta et al., 2006). Mediante este índice es posible comparar el peso ecológico de cada especie dentro del ecosistema. La obtención de índices de valor de importancia similares para las especies indicadoras, sugieren la igualdad o por lo menos la semejanza del rodal en su composición, estructuras, sitio y dinámica (Lamprecht, 1990).

TABLA 1. Diferencias biofísicas entre las parcelas

\begin{tabular}{|c|c|c|c|c|c|c|c|c|c|c|}
\hline & \multicolumn{4}{|c|}{ Sitio 1- Encinar menos antropizado } & \multicolumn{4}{|c|}{ Sitio 2- Encinar más antropizado } \\
\hline Parcelas & 1 & 2 & 3 & 4 & 5 & 6 & 7 & 8 & 9 & 10 \\
\hline Antropización & & & & & & & & & \\
\hline
\end{tabular}


Los diámetros obtenidos fueron agrupados en clases diamétricas con cinco centímetros de amplitud, a partir de las cuales se construyó un histograma de frecuencias.

\section{Estructura vertical}

La estructura vertical se describió tomando en consideración los tres estratos principales por los que están formados estos bosques (arbóreo, arbustivo y herbáceo) y los helechos, según la metodología descrita por Del Risco y González (2004) para el ecosistema pino-encino citado por González, Sánchez y Figueroa (2012). Siguiendo la recomendación de Delgado, Finegan, Zamora y Meir (1997) para este tipo de estudio, se registraron los individuos con más de $5 \mathrm{~cm}$ de $\mathrm{d}_{1,30 \mathrm{~m}}$.

- Herbáceo. Plantas menores de $1 \mathrm{~m}$ de altura.

- Arbustivo. Arbustos y arbolitos entre $1 \mathrm{~m}$ y $5 \mathrm{~m}$ de altura.

- Arbóreo. Árboles mayores de $5 \mathrm{~m}$ de altura.

- Helechos. Diferentes tipos de helechos terrestres, los que son muy importantes en la composición de los pinares como indicadores, por eso se han extraído de los estratos a los que corresponden según la altura, pues se encuentran en los tres estratos antes descritos, principalmente en el herbáceo.
- Lianas. Diferentes tipos de bejucos o lianas, los que pueden dar idea del estado sucesional en que se encuentra el tipo de bosque.

- Epífitas. Diferentes especies que viven sobre otras, tomándolas solo como sostén, pero sin parasitarlas ni causarles daños, las que a veces juegan un importante papel indicador sobre todo de la humedad ambiental.

\section{Resultados}

\section{Diversidad alfa $(\alpha)$}

El estudio florístico reveló para los dos encinares estudiados un total de 39 especies, pertenecientes a 25 familias (Tabla 2). La familia más representada fue Melastomataceae, con cuatro especies, seguida de las familias Fabaceae, Rubiaceae y Sapindaceae, con tres especies cada una. Cuando se analiza la riqueza de especies mediante las curvas de rarefacción basadas en el número de muestras (Fig. 1), como el número máximo es el mismo para cada encinar, las comparaciones se realizan en la asíntota. Como los intervalos de confianza de 95\% para la riqueza promedio de especies no se sobreponen entre ambas áreas, entonces existen diferencias significativas entre su riqueza.

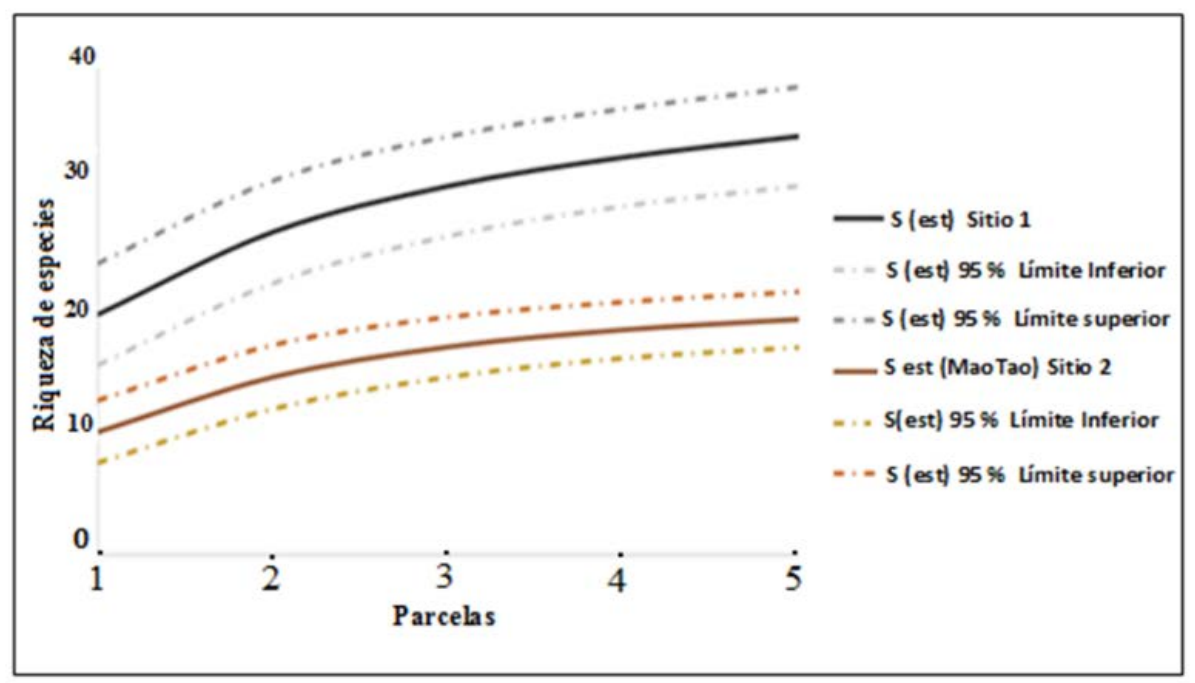

FIGURA 1. Curvas suavizadas de acumulación de la riqueza observada (curvas de rarefacción basadas en muestras) para cada encinar estudiado.

Sitio 1- Encinar menos antropizado, Sitio 2- Encinar más antropizado. 
TABLA 2. Listado Florístico de encinares naturales en las Alturas de Pizarras del Municipio de Viñales.

\begin{tabular}{|c|c|c|c|}
\hline No & Nombre científico & Nombre vulgar & Familia \\
\hline $\begin{array}{l}1 \\
2\end{array}$ & $\begin{array}{c}\text { Hybanthus havanensis Jacq. } \\
\text { Bambusa vulgaris J.C. }\end{array}$ & $\begin{array}{c}\text { Jia } \\
\text { Bambú }\end{array}$ & $\begin{array}{l}\text { Violaceae } \\
\text { Poaceae }\end{array}$ \\
\hline $\begin{array}{l}3 \\
4\end{array}$ & $\begin{array}{c}\text { Myrica cerífera Sw. } \\
\text { Alibertia edulis (L.C.Rich.) }\end{array}$ & $\begin{array}{c}\text { Arraigan } \\
\text { Pitajoní hembra }\end{array}$ & $\begin{array}{l}\text { Myricaceae } \\
\text { Rubiaceae }\end{array}$ \\
\hline 5 & Alophylus cominia Sw. & Palo de caja & Sapindaceae \\
\hline 6 & Andira inermis Sw. & Yaba & Fabaceae \\
\hline 7 & Andropogum virginicus $L$. & Pajón hembra & Poaceae \\
\hline 8 & Bursera Simaruba L. & Almacigo & Burseraceae \\
\hline 9 & Bysinima crassifolia (L.) HBK & Peralejo & Malpighiacea \\
\hline 10 & Calophyllum antillanum Bisse & Ocuje & Cluseaceae \\
\hline $\begin{array}{c}11 \\
12\end{array}$ & $\begin{array}{l}\text { Casearia hirsuta } S w \text {. } \\
\text { Chrysobalanus icaco } L \text {. }\end{array}$ & $\begin{array}{l}\text { Raspa lengua } \\
\text { Icaco }\end{array}$ & $\begin{array}{c}\text { Salicaceae } \\
\text { Chrysobalanaceae }\end{array}$ \\
\hline 13 & Citharexylum fruticosum Lin. & Canilla de venado & Verbenaceae \\
\hline 14 & Clidemia hirta (L)D.Don & Cordobán pelu & Melastomataceae \\
\hline $\begin{array}{l}15 \\
16\end{array}$ & $\begin{array}{l}\text { Clidemia neglecta D. Don } \\
\text { Clusea rosea Jaca }\end{array}$ & $\begin{array}{l}\text { Cordobán liso } \\
\text { Copey }\end{array}$ & $\begin{array}{l}\text { Melastomataceae } \\
\text { Clusiaceae }\end{array}$ \\
\hline 17 & Constegia xalapensis $D$. & Cordobán grande & Melastomataceae \\
\hline 18 & Cyathea arborea Smith. & Helecho & Cyatheaceae \\
\hline 19 & Cupania Glabra Sw. & Guara americana & Sapindaceae \\
\hline 20 & Davilla rugosa Poir. & Bejuco colorado & Dilleniaceae \\
\hline 21 & Didimopanax morototoni (Aubl).Dc & Yagrumon & Araliaceae \\
\hline 22 & Eugenia farameoides Sw. & Eugenia & Myrtaceae \\
\hline 23 & Faramea occidental Lin. & Cafetillo & Rubiacea \\
\hline 24 & Ficus abutilifolia (Miq.) & Ficus & Moraceae \\
\hline 25 & Guarea guidoni (L.) & Yamao & Meliaceae \\
\hline 26 & Hebestigma cubensis H.B.K & Frijolillo & Fabaceae \\
\hline 27 & Jambosa vulgaris DC. & Pumarrosa & Myrtaceae \\
\hline 28 & Matayba apetala (Marcf.) & Macurije & Sapindaceae \\
\hline 29 & $\begin{array}{c}\text { Pinus caribeae Morelet var caribaea } \\
\text { Barret y Golfari }\end{array}$ & Pino Macho & Pinaceae \\
\hline 30 & Pinus tropicalis Morelet & Pino hembra & Pinaceae \\
\hline 31 & $\begin{array}{l}\text { Pithecellobium berterianum (DC.) } \\
\text { Benth. }\end{array}$ & Moruro & Fabaceae \\
\hline 32 & Quercus cubana A.Rich & Encino & Fagaceae \\
\hline 33 & Roigella correifolia Griseb. & Jazmin del pinar & Rubiaceae \\
\hline 34 & Roystonia regia (Kunth) & Palma real & Arecaceae \\
\hline 35 & Sida acuta Burm. & Guisazo de caballo & Malvaceae \\
\hline 36 & Sphaeropteris myosuroides (Liebn.) & Rabo de Mono & Cyatheaceae \\
\hline 37 & Tabernaemontana amblyocarpa $L$. & Huevo de gallo & Apocynaceae \\
\hline 38 & Tetrazygia bicolor(Mill.)Cogn & Cordobancillo & Melastomataceae \\
\hline 39 & Xylopia aromatica (Lam.)Mart & Malagueta & Annonaceae \\
\hline
\end{tabular}


En la figura 2 puede observarse el comportamiento de la riqueza de especies por parcelas según los índices de diversidad. En el área menos antropizada se aprecia que las parcelas 1 y 2 presentan los valores más altos del índice de riqueza de especies; sin embargo, las parcelas 4 y 5 presentan los valores más bajos. Se aprecia que las parcelas que representan el mayor índice de abundancia de especies son la 1 y la 3 , las parcelas 4 y 5 presentan los valores más bajos del índice evaluado. De forma general, en el encinar menos antropizado se observa una mayor riqueza de especies.

\section{Diversidad beta( $\beta$ )}

El ordenamient de las parcelas con el empleo del análisis de jerarquización (Fig. 3) identifica que las especies se agruparon fundamentalmente en tres grupos: un solo grupo por su similitud en el encinar menos antropizado, mientras que en el área más antropizada se aprecia que se forman dos grupos bien diferenciados: uno con las parcelas 1, 2, 3 y el otro con las parcelas 4 y 5 .

\section{Estructura horizontal}

La distribución diamétrica de la vegetación en el encinar menos antropizado (Fig. 4) se asemejó a la forma típica de una $\mathrm{J}$ invertida, representativa de un bosque natural (Sidoruk, 2000). La mayoría de los individuos se concentran en las clases diamétricas inferiores, formando parte de la regeneración natural. En el encinar más antropizado se observa una distribución diamétrica diferente. La distribución por clases diamétricas de este bosque refleja un bosque secundario en proceso de recuperación, donde la regeneración ha sido en ciclos; en general son bosques más heterogéneos.

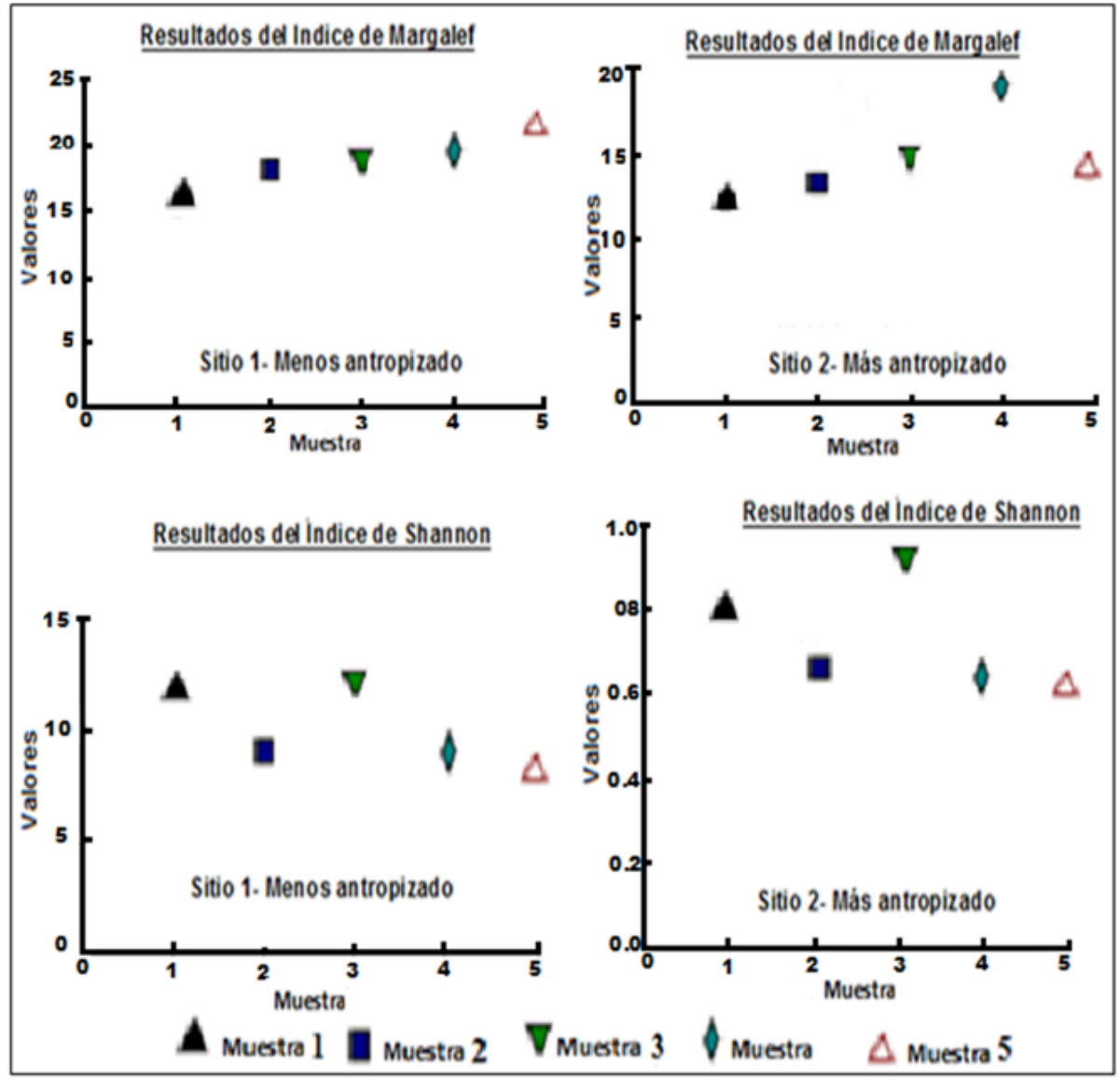

FIGURA 2. Riqueza de especies en los encinares estudiados. 


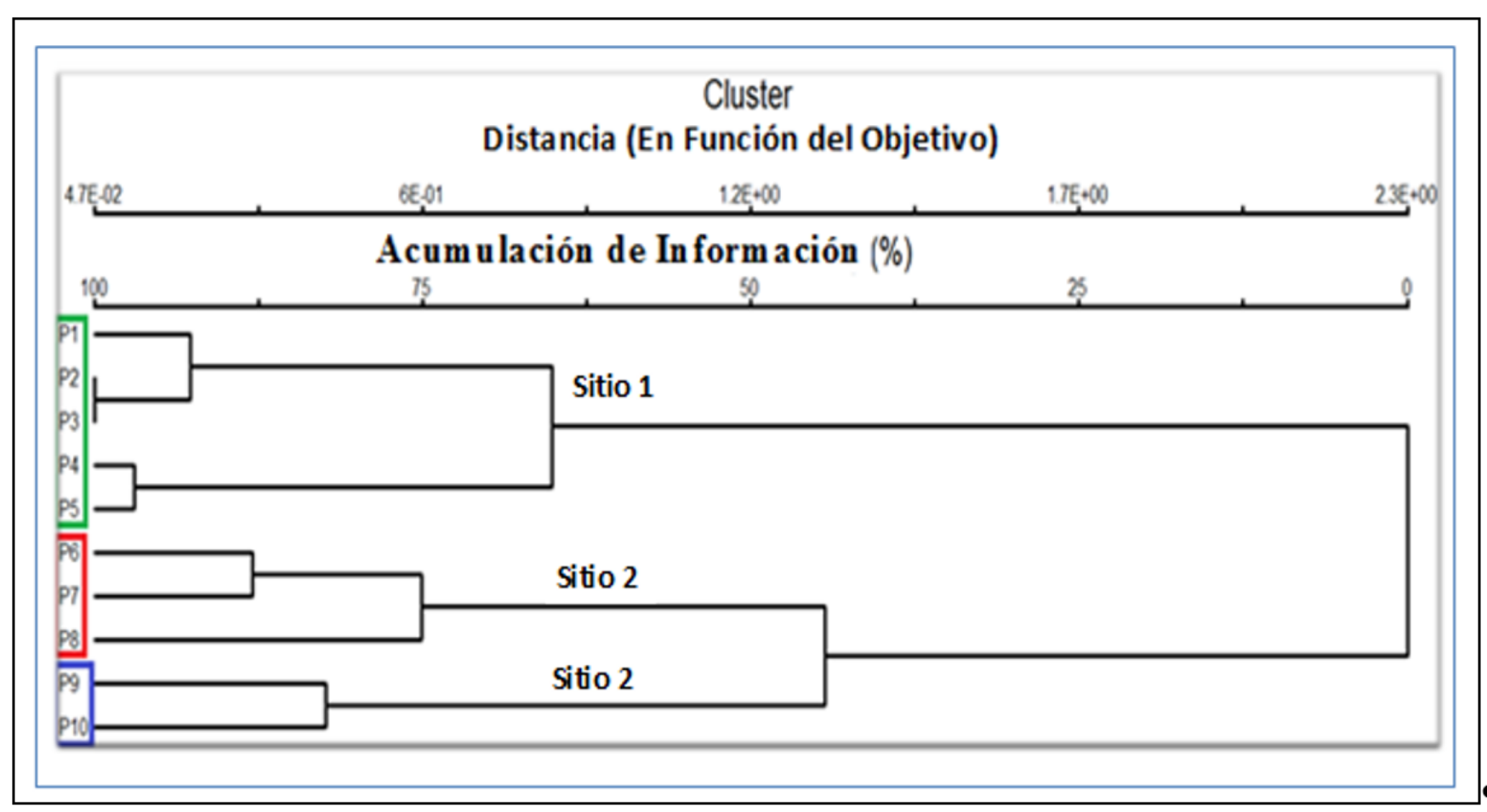

Similitud entre parcelas en el área menos antropizada
Similitud entre parcelas en

el área más antropizada
Similitud entre parcelas en

el área más antropizada

FIGURA 3. Dendrograma de clasificación de las parcelas para los encinares estudiados.

Sitio 1- Encinar menos antropizado. - Sitio 2- Encinar más antropizado.

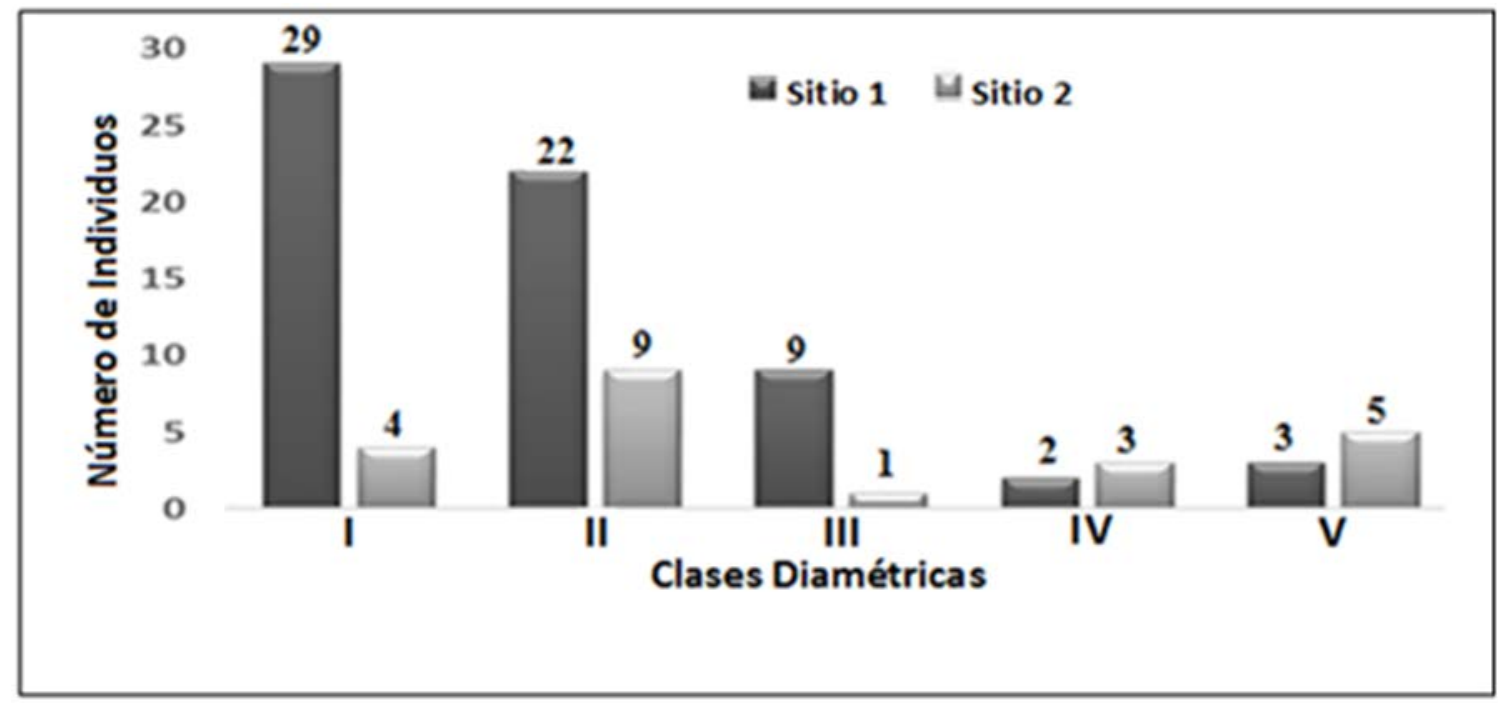

FIGURA 4. Distribución por clases diamétricas para las áreas estudiadas.

Sitio 1- Encinar menos antropizado, Sitio 2- Encinar más antropizado 
Índice de valor de importancia ecológica (IVIE)

Para tener una visión más amplia de la importancia de cada especie en conjunto en ambos encinares, en la figura 5 se ordenan las especies de acuerdo con los valores del IVIE; las 20 primeras en forma decreciente y el conjunto restante lo constituye una sola categoría denominada especies raras u otras especies según Melo y Vargas (2003). Esto permitió obtener una primera aproximación del valor de la diversidad y de la heterogeneidad del bosque. Se observó pobre presencia de frutos de encino en el suelo, afectaciones en su estructura, escasa regeneración natural y un elevado pastoreo de cerdos en el área (Fig.6).

\section{Estructura vertical de la vegetación}

Cuando se analiza la formación de encinares según el número de individuos por estrato (Fig.7), la estructura del Q. cubana en el encinar menos antropizado muestra la inexistencia de una secuencia demográfica creciente al reducirse el número de juveniles, con lo cual se afecta el reclutamiento. Al respecto, en el encinar más antropizado (Fig.8), la composición del encino por estrato muestra la tendencia a una curva en forma de J invertida.

\section{DIISCUSIÓN}

\section{Diversidad alfa $(\alpha)$}

En cuanto a la composición de especies, Cascante y Estrada (2000) obtuvieron un resultado similar en el estudio de la composición florística y estructura de un encinar en la zona húmeda premontana en el valle central de Costa Rica. Estos mostraron que en un área de aproximadamente 2.40 ha (cinco parcelas de $100 \mathrm{~m}^{2}$ ), se observó 85\% del total de especies del estudio y que el aumento en la intensidad del muestreo no provee un aumento significativo en la riqueza de especies.

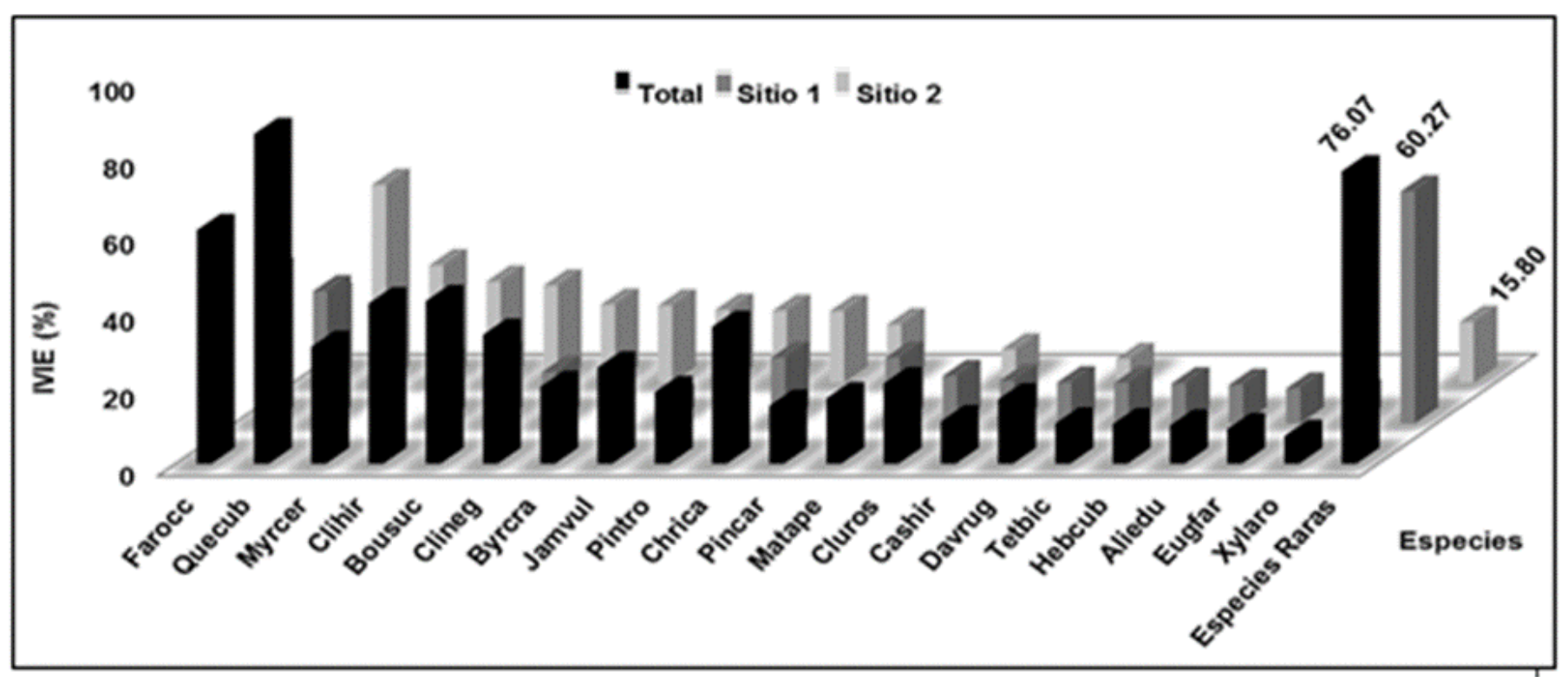

FIGURA 5. Índice de valor de importancia ecológica del encinar.

Sitio 1- Encinar menos antropizado, Sitio 2- Encinar más antropizado.

Faramea occidentalis (Farocc), Quercus cubana (Quercub), Myrica cerifera (Myrcer), Clidemia hirta (Clihir), Bursera Simaruba (Bousuc), Clidemia neglecta (Clineg), Bysinima crassifolia (Byrcra), Jambosa vulgaris (Jamvul),Pinus tropicalis (Pintro), Chrysobalanus icaco (Chrica), Pinus caribeae (Pincar), Matayba apetala (Matape), Clusea rosea (Cluros), Casearia hirsuta (Cashir), Davilla rugosa (Davrug), Tetrazygia bicolor (Teebic), Hebestigma cubensis (Hebcub), Alibertia edulis (Aliedu), Eugenia farameoides (Eugfar), Xylopia aromatica (Xylaro), Especies raras. 


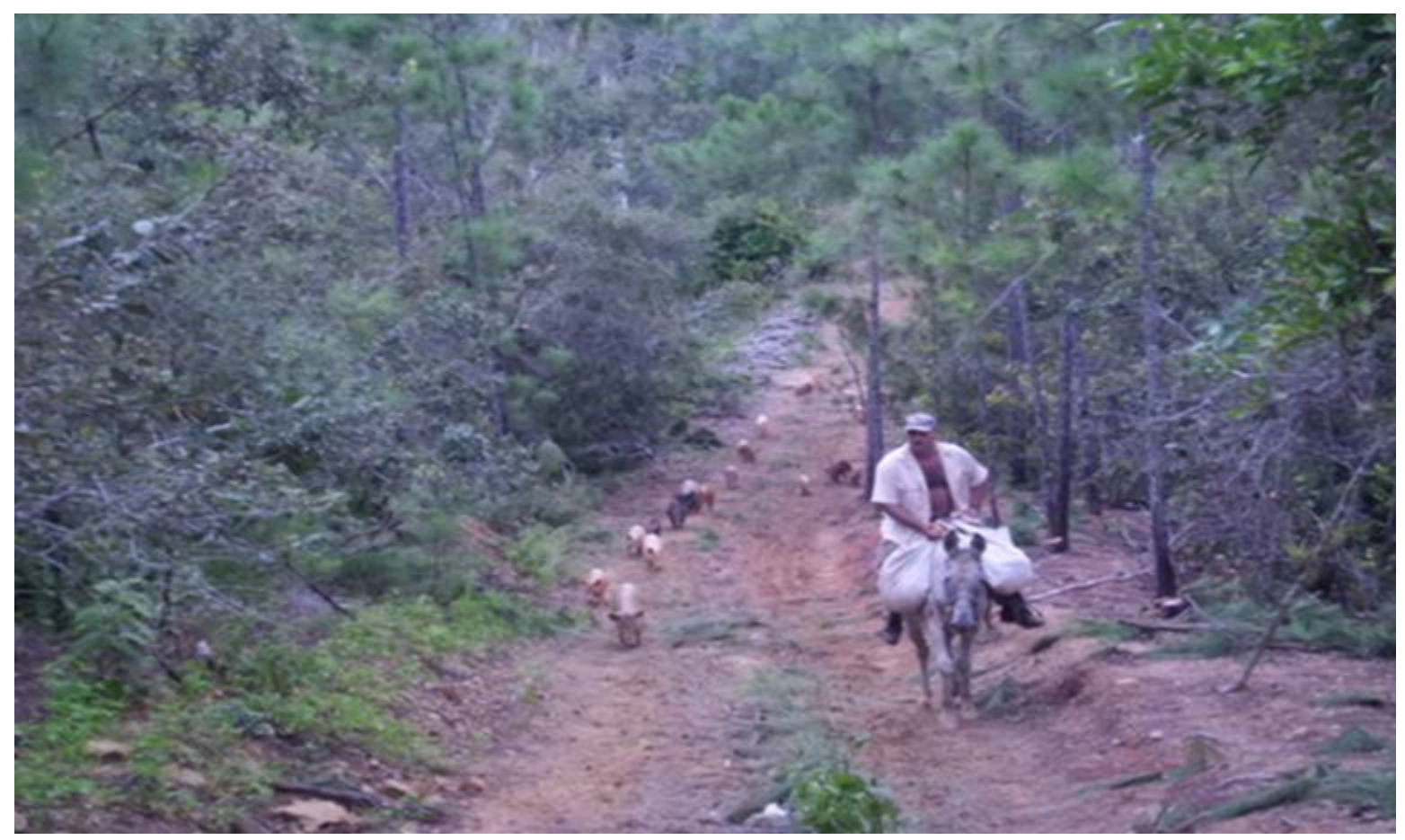

FIgURA 6. Silvopastoreo en encinares en la empresa Agroforestal La Palma.

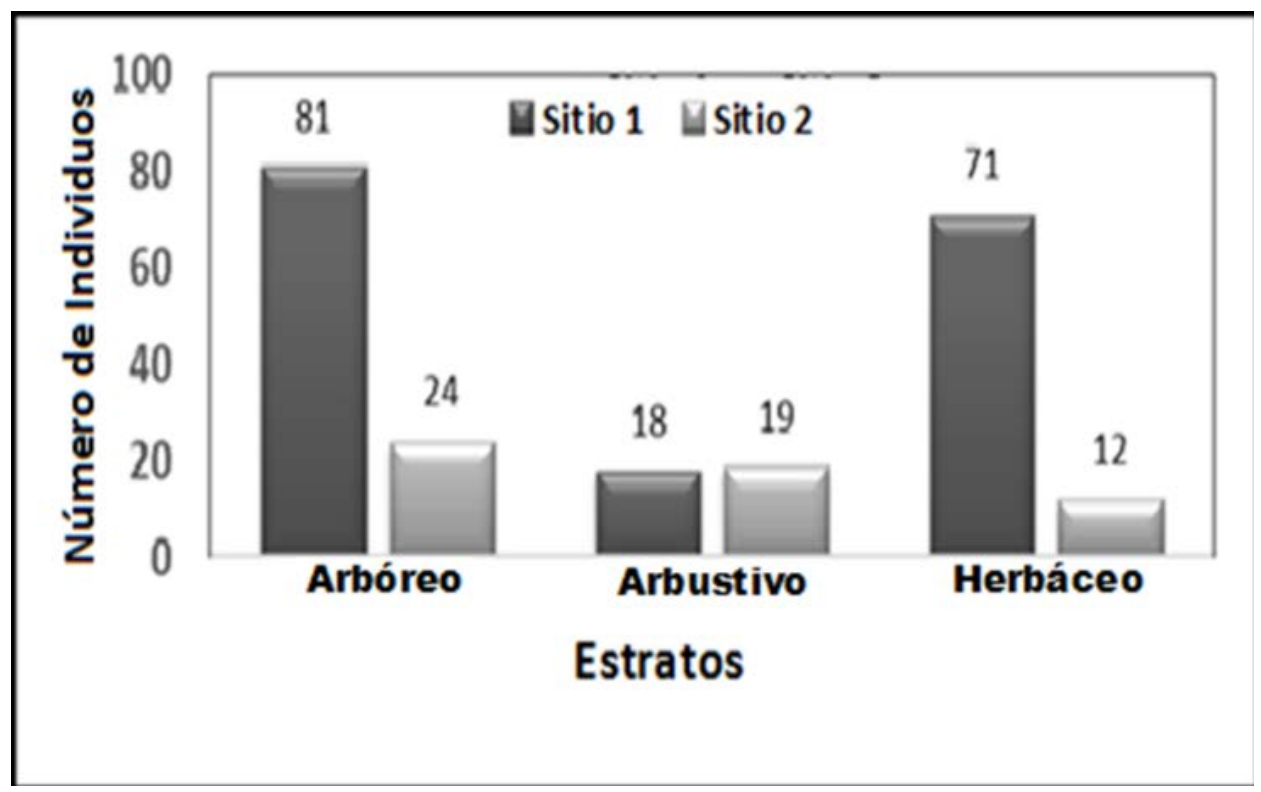

FIGURA 7. Estructura vertical de los encinares por estratos. Sitio 1- Encinar menos antropizado, Sitio 2- Encinar más antropizado 


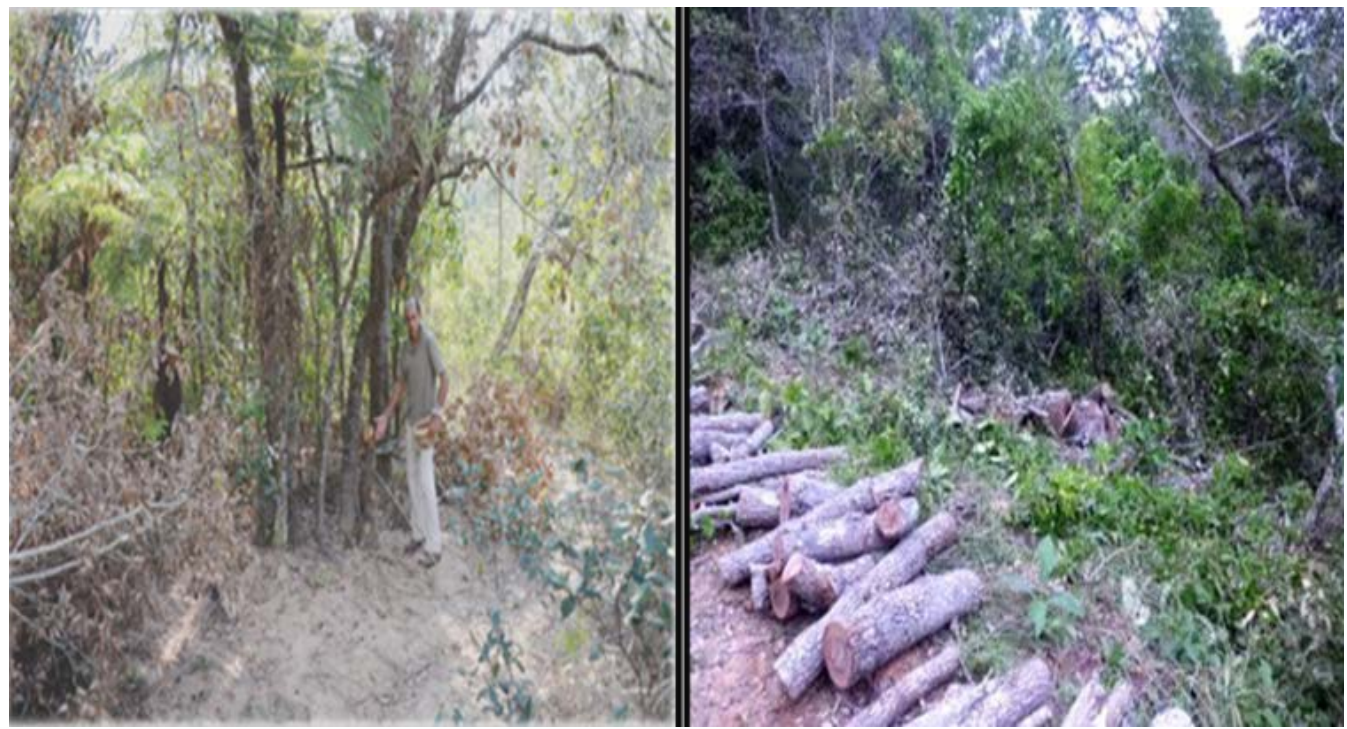

FIGURA 8. Encinar más antropizado.

Según Delgado et al. (1997), la determinación de la composición florística de los bosques (familias, especies) ayuda a caracterizar las comunidades y generar información acerca de la dinámica de los bosques naturales y su respuesta a diferentes regímenes de perturbación. Estos resultados se corresponden con los encontrados por González et al. (2012) en estudios realizados en ecosistemas de pino-encino en Alturas de Pizarras de Viñales, mostrándose que la sistematicidad de tratamientos silvícolas inadecuados en este ecosistema, atentó contra la conservación de la diversidad florística natural y la ocurrencia de cambios en las condiciones ecológicas óptimas para el desarrollo de especies nativas de esta formación.

En el área se encuentran representadas cinco de las diez familias registradas por Acevedo y Strong (2012) como las de mayor diversidad de taxa en las Antillas: Euphorbiaceae, Fabaceae, Melastomataceae, Poaceae, Rubiaceae y Urticaceae, lo que resalta el valor ecológico y el potencial de conservación de la vegetación montañosa analizada.

\section{Diversidad beta $(\beta)$}

En el análisis de jerarquización del encinar menos antropizado se identificó un solo grupo por su similitud, con más del $60 \%$ de similitud (sitio 1) formado por las parcelas de la 1 a la 5. De estos resultados se infiere que sea consecuencia de la cercanía de las áreas al asentamiento poblacional "Los Jazmines" y la cercanía a la carretera principal que va a hacia el pueblo de Viñales, lo que favorece el aprovechamiento de los árboles con diámetros superiores en toda el área, para ser empleados como horcones u otras obras que demanden estas dimensiones.

El comportamiento de la similitud de las parcelas en el área más antropizada, refleja la formación de dos grupos bien diferenciados. El primero, formado por las parcelas 6, 7 y 8 con más del 75\% de similitud, se encuentra ubicado en la parte más plana de la meseta, alejado de la carretera, pero cerca de la comunidad rural nombrada "La Cuadra", perteneciente al Consejo Popular Los Jazmines; en él se observa afectación por la tala de los árboles y el sobrepastoreo del ganado porcino, afectando considerablemente la composición y estructura del suelo como se mostró en párrafos anteriores, afectando el desarrollo de la regeneración natural del Q. cubana e influyendo en gran medida sobre la estructura y composición del ecosistema. El segundo grupo, igualmente con más de $75 \%$ de similitud, está integrado por las parcelas 9 y 10. Estas últimas parcelas se ubicaron en la parte más distante del asentamiento, coincidiendo con la parte más 
baja de la cuenca; esto lo hace un sitio muy difícil para la tala y acarreo de árboles, por lo que conserva un estrato arbóreo con buenos fenotipos, específicamente de encinos.

Sin embargo, en el estrato herbáceo no se observa un comportamiento similar, pues al estar estas parcelas colindantes con el río La Majagua, se crearon las condiciones para que los cerdos pastaran y hozaran en demasía, estimulados por la humedad y la acumulación de bellotas, viéndose afectada la regeneración natural.

\section{Estructura horizontal}

El comportamiento observado al determinar el índice de riqueza de especies (Margalef) en el encinar más antropizado, está relacionado con el hecho de que la parcela es la más cercana al asentamiento poblacional y a la carretera, por lo cual resulta fácil adentrarse en el bosque y extraer los árboles dominantes de encino quedando el estrato arbóreo abierto dando paso al desarrollo de las especies acompañantes.

En la parcela 1, situada en la parte baja de la cuenca, más distante, sucede lo contrario, resultando más difícil la tala de los árboles dominantes, por lo que el estrato arbóreo queda dominado por árboles de encino de mayor porte, $\mathrm{y}$ esto regula la estructura bajo el dosel. Es de resaltar que en estas parcelas González et al. (2016) seleccionaron 10 árboles fenotípicamente superiores.

Como se aprecia en los anteriores resultados, hay una influencia significativa de los disturbios presentes: tala ilícita de árboles y arbustos y sobre pastoreo intensivo y descontrolado, en la estructura y composición florística de esta formación natural. Al respecto, Timilsina, Ross y Heinen (2007) plantean que la estructura, composición y función son atributos importantes de los ecosistemas boscosos y cambian en respuesta a clima, topografía, suelos y disturbio. Estos factores de conjunto con el proceso sucesional de la vegetación, son responsables de la variación en los atributos del bosque a escala local y de paisaje, al producir heterogeneidad espacial.

Al respecto, Pickett y White (1985) plantean que las alteraciones que ocurren en los ecosistemas por causas antropogénicas pueden ser irreversibles o persistentes por períodos prolongados. Algunos de los cambios pueden percibirse como positivos, ya que favorecen a especies utilizadas como recursos, o crean condiciones favorables para los seres humanos.

La estructura horizontal observada en el encinar menos antropizado, sugiere el aseguramiento de la viabilidad de las poblaciones de las especies, ya que Garzón (2001) y Tenorio, Solano y Castillo (2009) informan que, con el tiempo, la regeneración pasa a ocupar las clases diamétricas mayores, renovando así las poblaciones y la posibilidad ante un manejo silvícola con criterios sostenibles de una recuperación de esta formación. Probablemente este hecho ocurrió debido al criterio de inclusión adoptado (individuos con diámetros a partir de los $5 \mathrm{~cm}$ ) que, según Narvaes, Brena y Longhi (2005), favorece el muestreo de los individuos en fase juvenil. La concentración de individuos de menor diámetro sugiere que la vegetación se autosustenta, ya que los mismos pertenecen a la regeneración natural y, según López, Ramírez y Zamora (2012), son capaces de establecerse durante los primeros años. Es observable que, conforme aumentan las clases diamétricas, disminuye el número de individuos producto de la competencia intra e interespecífica. La escasez y ausencia de árboles en las clases diamétricas superiores pueden relacionarse, además, con la corta de los mejores fenotipos.

\section{Índice de Valor de Importancia Ecológica}

El índice de valor de importancia ecológica del encinar más antropizado muestra que la primera posición la ocupa $Q$. cubana A.Rich, mientras que en el encinar menos antropizado, la primera posición la ocupa la especie Faramea occidentalis Lin., con Q. cubana A. Rich en segundo lugar. Al analizar el número de individuos por estrato del encinar menos antropizado, la estructura del $Q$. cubana muestra la inexistencia de una secuencia demográfica creciente al reducirse el número de juveniles, con lo cual se afecta el reclutamiento. Según Clark et al. (1999), este comportamiento es conocido como la inclusión de nuevos 
individuos a la población, a las clases de edades superiores. La estructura del Q. cubana mostró inestabilidad en la abundancia de los individuos. Las plántulas estuvieron presentes en los tres estratos, encontrándose un número reducido de individuos en el estrato arbustivo con solo $10 \%$, lo que denota la baja capacidad reproductiva de los árboles; sin embargo, en el estrato herbáceo se concentró $42 \%$ de los individuos, indicando dificultades con el paso de los individuos de plántulas a juveniles.

El comportamiento observado en el IVIE, respecto a la ubicación de F. occidentalis en la primera posición en importancia, se debe a la alta frecuencia y fundamentalmente a su elevada abundancia en el estrato herbáceo y arbustivo. La segunda posición ocupada por $\mathcal{Q}$. cubana es el resultado de la cercanía del sitio 1 al asentamiento poblacional Los Jazmines: se encontraron evidencias de que los mejores fenotipos han sido talados para ser utilizados como horcones, para la construcción de viviendas y casas, y las ramas en la fabricación de instrumentos agrícolas como rastras para ser acarreadas por bueyes y para la fabricación de carbón vegetal o uso directo como leña, debido a su alto poder calorífico. Esta tala deja abierto el dosel, lo que favorece el crecimiento de las especies secundarias.

La primera posición de Q. cubana, al analizar el IVIE en el encinar más antropizado, se debe a su mayor presencia en el estrato arbóreo, dominado por ejemplares de gran porte. La presencia de estos fenotipos se debe a lo inaccesible del área, lo que impide que estos puedan ser cortados. Estos encinares se encuentran localizados en la parte baja de la cuenca, además de estar muy alejados del asentamiento poblacional.

En el área más antropizada se observaron arbustos cortados para ser empleados como postes de cerca de potreros para la cría extensiva de cerdos. El finquero encargado del pastoreo de los cerdos plantea que, en la época de fructificación de los encinos, los campesinos de la zona utilizan el área para la cría ilegal, aumentando de forma considerable y desmedida la carga de cerdos en el sitio. Esto trae como resultado el agotamiento de los frutos, con la consiguiente degradación de los suelos y afectaciones en la regeneración natural, viéndose afectada la sucesión natural (Izquierdo et al., 2013) y (Santana et al., 2015).

\section{Estructura vertical de la vegetación}

Al observar la composición del encino por estrato en el encinar más antropizado, se aprecia la tendencia a una curva en forma de J invertida. En este sentido, Primack, Rozzi, Feisinger, Dirzo, y Massardo (2001) exponen que una población típicamente estable posee una distribución de edades con una relación característica de juveniles, adultos jóvenes y adultos viejos, o sea que la ausencia o baja representatividad de cualquier clase de edad, especialmente juveniles, señala hacia potenciales poblaciones en decline. La baja presencia de jóvenes puede ser indicativo de una población estable e incluso en expansión. Este tipo de distribución surgiere una alta tendencia a la heterogeneidad (Melo y Vargas, 2003).

La frecuencia de la primera clase o estrato arbóreo es mayor que la segunda, estrato arbustivo, lo que revela un bosque donde predomina un dosel medio con escasa regeneración natural.

De acuerdo con estos resultados, puede afirmarse que todas las localidades de un modo u otro han sido perturbadas por la tala selectiva de los individuos de encino de mayor diámetro, alterando la estructura diamétrica de estos bosques, por lo que predominan árboles de diámetro pequeño.

Al respecto, González y Sotolongo (2007) recalcan que en cualquier región forestal, las perturbaciones, sean de una clase u otra, se encuentran alterando continuamente el curso de la sucesión forestal e iniciando una sucesión secundaria.

Los resultados obtenidos, referente al efecto de la antropización en el bajo logro de la regeneración natural en estos encinares, coinciden con lo planteado por Jeffrey (2015), al dejar claro que la mayoría de las semillas de los encinos que maduran antes del tiempo, donde de forma natural existen las condiciones para que se logren, no tienen ninguna oportunidad de sobrevivir hasta las primeras 
lluvias. Este autor plantea que las semillas que caen en el mes de octubre no sobreviven hasta mayo del siguiente año, debido a que el mayor por ciento es consumido por roedores o por cerdos o pierden la facultad germinativa.

Por otro lado, los ecosistemas cubanos en los que se desarrolla Quercus son suelos pobres y degradados por el manejo. Según estudios realizados por Jeffrey (2015), los encinos también son importantes para la flora silvestre y generan mucha materia orgánica, en ocasiones son de las pocas especies que se desarrollan en sitios con suelos degradados y con poca materia orgánica. Las ramas actúan como substrato para el desarrollo de variadas epífitas, como orquídeas (Orchidaceae), bromelias (Bromeliaceae) y muérdagos (Viscaceae). Entre los hongos, algunas especies dependen de los encinos, como las micorrizas, y otras llevan alguna relación simbiótica distinta con plantas de este género. Como puede apreciarse, los estudios florísticos y estructurales de la vegetación son de indiscutible importancia para el conocimiento de los bosques y su funcionabilidad, así como para la toma de decisiones con respecto al manejo que debe aplicarse en ellos sin alterar el equilibrio estructural de forma brusca y simulando la trayectoria sucesional intrínseca de todo bosque frente a las perturbaciones de cualquier tipo. López et al. (2012) demostraron, en estudios realizados en ecosistemas naturales; que particularmente la deforestación y la fragmentación de hábitat ocasionan alteraciones significativas de distribución y abundancia de organismos. El estudio y comprensión de este proceso en los remanentes de bosques puede ser de importancia crucial para el manejo y conservación del sistema natural.

\section{CONCLUSIONES}

La riqueza de especies evidencia una alta diversidad florística asociada al encinar natural en la zona de estudio. Se inventariaron un total de 38 especies asociadas a los encinos, pertenecientes a 25 familias.

El nivel de antropización (silvícola y pastoril del rebaño porcino) tuvo una marcada influencia en la estructura y composición de los encinares naturales en la zona estudiada.
La condición de Quercus cubana A. Rich como especie dominante en los encinares, con una estructura, la convierte en vulnerable ante disturbios naturales y antrópicos potenciales, evidenciando una débil habilidad competitiva

\section{RECONOCIMIENTOS}

La presente investigación fue apoyada por los especialistas de la Estación Experimental Agroforestal de Viñales: Segundo Curbelo, Pedro Friol y Barboro Cabrera. De igual forma se agradece la ayuda brindada por el Dr. C Yuris Rodríguez Matos del Departamento Forestal de la Universidad de Guantánamo, Cuba.

\section{REFERENCIAS}

Acevedo, P. \& Strong, M. (2012). Catalogue of Seed Plants of the West Indies. Smithsonian Contribitions to Botany, 98, 1-1192. doi: 10.5479/si.0081024X.98.1

Acosta, V. H., Araujo, P. A., \& Iturre, M. C. (2006). Caracteres estructurales de las masas. Serie Didáctica No. 2. Universidad Nacional de Santiago del Estero, Santiago del Estero, Argentina.

Cascante, M. A. \& Estrada, Ch. A. (2000). Composición florística y estructura de un bosque búmedo pre montano en el Valle Central de Costa Rica. San José, Costa Rica: Departamento de Historia Natural, Museo Nacional de Costa Rica.

Clark, J. S., Beckage, B., Camill, P., Cleveland, B., Hillerislambers, J., Lichter, J., Mclachlan, J., Mohan, J., \& Wyckoff, P. (1999). Interpreting recruitment limitation in forests. American Journal of Botany, 86(1), 1-16.

Colwell, R. K. (2011). Estimates: Statistical estimation of species richness and shared species from samples (version 9) [software de cómputo]. Recuperado de http://purl.oclc.org/estimates.

Delgado, D., Finegan, B., Zamora, N., \& Meir, P. (1997). Efectos del aprovechamiento forestal y el tratamiento silvicultural en un bosque búmedo del noreste de Costa Rica, cambios en la riqueza y composición de la vegetación. Colección Manejo Diversificado de Bosques Naturales 298. CATIE.

Finegan, B., Palacios, W., Zamora, N., \& Delgado, D. (2001). Ecosystem-level forest biodiversity and sustainability assessments for forest management. En R. Raison, A. Brown, \& D. Flinn (Eds.), Criteria and indicators for sustainable forest management (pp. 341-378). Viena, AT: IUFRO.

Garzón, H. E. (2001). Estudio poblacional de guayacán real (Guaiacum sanctum L.) en el Parque Nacional Palo Verde y en Las Delicias de 
Garza, Guanacaste. Maestría en Desarrollo Rural.. Escuela de Ciencias Ambientales. Costa Rica.

González, E. \& Sotolongo, R. (2007). Ecología forestal. La Habana: Editorial Félix Varela.

González, M., Sánchez, O., \& Figueroa, C. (2012). Restauración de la biodiversidad florística en ecosistemas degradados de Pinus caribaea Morelet. Revista Forestal Baracoa, 31(1), 17-23. 2078-7235.

González, M., Fidelmant, M., Lebbet, M., Miñoso, Y., Sanches, O., , \& Friol, P. (2016). Inicio de un programa de manejo silvícola de Quercus cubana A. Rich. en la provincia Pinar del Río, Cuba. Fase I. Memorias del Simposio Internacional sobre Manejo Sostenible de los Recursos Forestales. Cuba: Universidad de Pinar del Río.

Hernández, A. J., Ascanio, M. O., Carrera, R. A., \& Morales, B. N. (2003). Nuevos aportes a la clasificación genética de los suelos en el ámbito nacional e internacional. Habana, Cuba: Instituto de Suelos-Ministerio de la Agricultura-Agrinfor.

Izquierdo, K., Pimentel, C. A., \& Márquez, L. (2013). Adaptación de plántulas reintroducidas de Quercus cubana, endémico de Cuba Occidental, en su medio natural del bosque de encinos de Manuel Lazo, Sandino, Pinar del Río. Revista Electrónica Avances, $\quad 4(1)$. Recuperado de http://revistaecovida.upr.edu.cu/index.php/ecovida/articl e/view/50/html

Jeffrey, R. (2015). Los encinos: importantes elementos de la biodiversidad latinoamericana. Recuperado de http://jrbacon.homtestead.com/jeff.html

Lamprecht, H. (1990). Silvicultura en los Trópicos. Los ecosistemas forestales en los bosques tropicales y sus especies arbóreas posibilidades y métodos para un aprovechamiento sostenido. República Federal de Alemania: Eschborn (Germany) GTZ.

López, L., Ramírez, Y., \& Zamora, Y. (2012). Evaluación de la diversidad florística en cuatro bosques de la zona amortiguadora del parque nacional natural los nevados. Boletín cientifico. Centro de Museos. Museo de Historia Natural, 16(1), 41-59.

Magurran, A. E. (1989). Diversidad ecológica y su medición. Barcelona, España: Ediciones Vedra.

McAleece, N., Gage, J. D., Lambshead, J., \& Patterson, G. (1997). Biodiversity Professional. Londres: The Natural History Museum \& The Scottish Association for Marine Science.

McCune, B. \& Mefford, M. J. (1999). Multivariate analysis of ecological data (Versión 4.0) [Software de cómputo] Glenenden Beach, Oregon, EUA.
Melo, O. \& Vargas, R. (2003). Evaluación ecológica y silvicultural de ecosistemas boscosos. Ibagué - Tolima. Colombia: Editorial Gente Nueva.

Moreno, C. (2001). Métodos para medir la Biodiversidad. México: Universidad Autónoma del Estado de Hidalgo.

Narvaes, I. S., Brena, D. A., \& Longhi, S. J. (2005). Estructura da regeneração natural em floresta ombrófila mista na floresta nacional de São Francisco de Paula, RS. Ciência Florestal, 15(4), 331-342.

Organización de las Naciones Unidas para la Alimentación y la Agricultura [FAO] (2014). Situación de los Bosques del mundo 2014. Roma: FAO.

Pennington, T. \& Sarukhán, J. (1998). Árboles tropicales de México. Manual para la identificación de las principales especies ( $\left.2^{\mathrm{a}} \mathrm{ed}\right)$. México, D.F.: Universidad Nacional Autónoma de México y Fondo de Cultura Económica.

Pickett, A. \& White, S. (1985). The ecology of natural disturbance and patch dynamics. EUA: Academic Press.

Primack, R., Rozzi, R., Feisinger, P., Dirzo, R., \& Massardo, F. (2001). Fundamentos de conservación biológica. Perspectivas latinoamericanas. México DF: Fondo de Cultura Económica.

Sánchez, C. (2007). Los belechos y licófitos de Cuba. La Habana: Científico Técnica.

Santana, I., Abeledo, C., Ferrer, R. M., González, M., \& Pimentel, J. (2015). Asociación cerdos criollo-encino en el municipio pinareño de la palma para la producción de carne ecológica de sabor especial. Revista Computadorizada de Producción Porcina, (2). Recuperado de http:/ /www.iip.co.cu/RCPP/222/222_10ISantana.pdf

Servicio Estatal Forestal [SEF] (2016). Encinares existentes en la Provincia de Pinar del Río. Dinámica 2016. Pinar del Río, Cuba: Servicio Estatal Forestal.

Sidoruk, V. (2000). Análise biométrica da regeneração natural de algunas espécies em uma Floresta Estacional Semidecidual localizada no Município de Cássia- MG. Curitiba. Tesis de maestría, Universidad Federal de Paraná. Paraná, Brasil.

Tenorio, M., Solano, C., \& Castillo, U. M. (2009). Evaluación de la composición florística y estructural en un bosque primario intervenido en la zona norte de Costa Rica. Revista Forestal Kurú, 6(16), 1-6.

Timilsina, D., Ross, M. S., \& Heinen, J. T. (2007). A community analysis of sal (Shorea robusta) forests in the western Terai of Nepal. Forest Ecology and Management, 241(1-3), 223-234. doi: 10.1016/j.foreco.2007.01.012 
Manuscrito recibido el 22 de noviembre de 2017

Aceptado el 5 de octubre de 2018

Publicado el 16 de octubre de 2019

Este documento se debe citar como:

González-Menéndez., M., Alonso-Torrens, Y., Urrutia-Hernández, I, Miñoso, Y., Santana, I., Luis-Suárez, J., \& González-Torres, C. M. (2019). Estructura y composición de encinares naturales en las Alturas de
Pizarras del Municipio de Viñales, Pinar del Río, Cuba. Madera y Bosques, 25(2), e2521667. doi: 10.21829/myb.2019.2521667

Madera y Bosques por Instituto de Ecología, A.C. se distribuye bajo una Licencia Creative Commons Atribución-NoComercialCompartirlgual 4.0 Internacional. 